\title{
An Interruption in the Highway: New Approach to Modeling Car Traffic
}

\author{
Amin Rezaeezadeh ${ }^{\star}$ \\ Physics Department \\ Sharif University of Technology \\ Tehran, Iran
}

Received: February 17, 2010

Accepted: February 9, 2011

\section{ABSTRACT}

A very common phenomenon in car traffic is investigated in this article. The problem is onedimensional. We find the wave equation of the traffic, and illustrate a simulation using Matlab 7.6

\section{INTRODUCTION}

Several articles and investigations have shown an increasing realization of the need for a scientific approach to various aspects of road traffic, a field that seemed formerly to lie at the periphery of physics. Nowadays, most of us experience car traffic during daily commutes, especially along ring roads around major cities. Here in Tehran, I have personally experienced large traffic jams along the Hemat highway involving thousands of cars. This stimulated me to investigate and model this type of car traffic, especially the conditions that lead to traffic jams. [1]

\section{THEORY}

\section{a. Parameter Definitions}

I will take a simple approach and focus on only one of the traffic lanes. In a traffic jam when traffic is heavy, cars will have limited opportunities to shift lanes, so this seems a reasonable first model. Let's consider this lane to be the "passing" or fastest lane.

To start our work, suppose there are $n$ cars driving at speed $V_{0}$ in one lane and that the safe distance [2] between cars at the rated speed is $I$ (Fig. 2). For convenience, let us neglect the delay in drivers' reaction times. An interruption is

*Amin67r@gmail.com

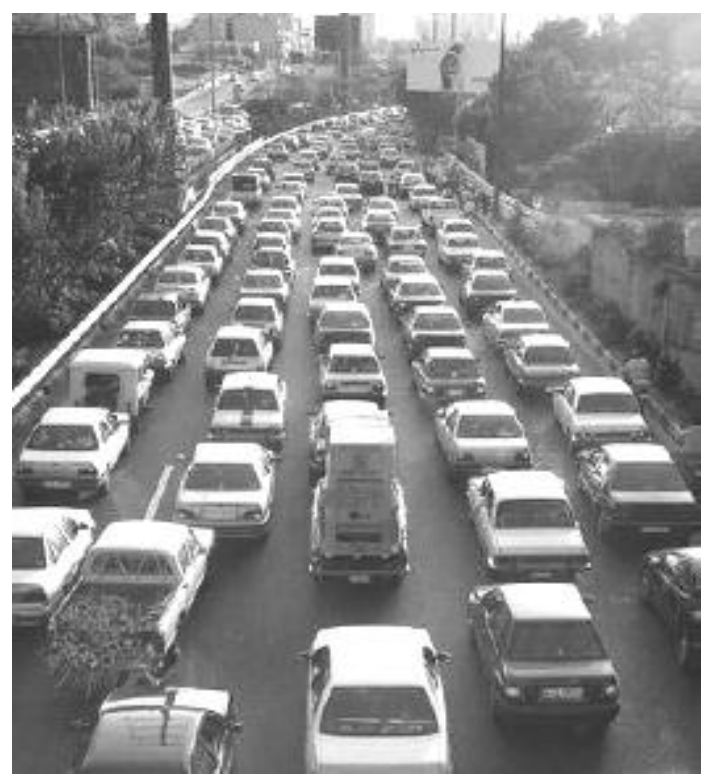

Figure 1. Hemmat Highway during rush hour.

applied to the system at $\mathrm{t}=0$ to a first car in traffic such that it suddenly stopsremember, we are assuming that drivers can control their cars without delays. Each following car should slow to a stop in a safe distance l' from the car in front of it.

\section{b. Speed Control Modeling}

As mentioned above, at rated speed $\left(V_{0}\right)$ all cars should maintain a safe distance I from the car in front of them. Since each car should stop in minimum distance $l$,' it is 
convenient to model speed control by a linear function of the distance between the two cars. That is, the velocity of car " $k$ " will depend on how far it is from car " $k-1$ " in front of it. Mathematically we can formulate this as,

$$
V_{k}(t)=V_{0}+\alpha\left(l_{k}(t)-l\right)
$$

Where $I_{k}$ is the distance between the $k^{\text {th }}$ car and the $(k-1)$ car immediately in front of it, and $\alpha$ is a constant which is determined by taking $V_{k}=0$ when $I_{k}=l$ '. We note that $\alpha$ can be different from car to car [3] though we will assume that all cars have the same safe distance, for convenience.

$$
0=V_{0}+\alpha\left(l^{\prime}-l\right)
$$

Therefore,

$$
\alpha=\frac{V_{0}}{l-l^{\prime}}
$$

From the definition of $I_{k}$ we have

$$
\frac{d l_{k}(t)}{d t}=V_{k-1}(t)-V_{k}(t)
$$

Substituting (3) into (1) we get a new equation for $V_{k}(t)$ :

$$
\frac{d V_{k}(t)}{d t}=\alpha\left(V_{k-1}(t)-V_{k}(t)\right)
$$

This, which has dimensions of acceleration, can be interpreted in terms of two effective forces [4]: motor reaction and a force proportional to the velocity (for example, a drag force due to air resistance).

\section{c. Propagating Wave}

Equation 4 describes a discrete wave that propagates over the cars. The information of the carrier wave is the interruption that the first car has made in the system. In order to find the velocity of the wave, suppose that the velocity of the $(\mathrm{k}-1)^{\mathrm{th}}$ car is equal to the velocity of the $k^{\text {th }}$ car after passing time $\tau$. That is,

$$
V_{k-1}(t)=V_{k}(t+\tau)
$$

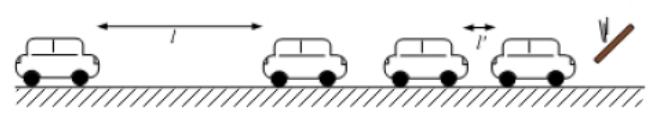

Figure 2. An interruptions at $t=0$ causes all cars to stop.

Assume $\tau$ is very small compared to $t$. Thus, it is possible to expand (5) to first order in $\tau$.

$$
V_{k}(t+\tau) \approx V_{k}(t)+\frac{d V_{k}(t)}{d t} \tau
$$

Substituting (6) into (4) we have,

$$
\frac{d V_{k}(t)}{d t}=\alpha\left(\frac{d V_{k}(t)}{d t} \tau\right)
$$

And therefore,

$$
\tau=\frac{1}{\alpha}=\frac{l-l \prime}{V_{0}}
$$

The velocity function of the first car $\left(V_{1}(t)\right)$ affects the motion of all other cars. Saying this another way, (4) has too many answers as a propagating wave. These answers are responses to $V_{1}(t)$ as an input to the car traffic system. Let us put all variables $V_{k}(t)$ in a vector $V$, i.e.

$$
V=\left(\begin{array}{c}
V_{2} \\
V_{3} \\
\vdots \\
V_{n}
\end{array}\right)
$$

Therefore, (4) is changed into an ordinary differential equation of vectors and matrices.

where,

$$
\dot{V}=A V+U
$$

and

$$
A=\left[\begin{array}{ccccc}
-\alpha & 0 & 0 & & 0 \\
\alpha & -\alpha & 0 & \ldots & 0 \\
0 & \alpha & -\alpha & & 0 \\
& \vdots & & \ddots & \vdots \\
0 & \ldots & 0 & \cdots & -\alpha
\end{array}\right]
$$




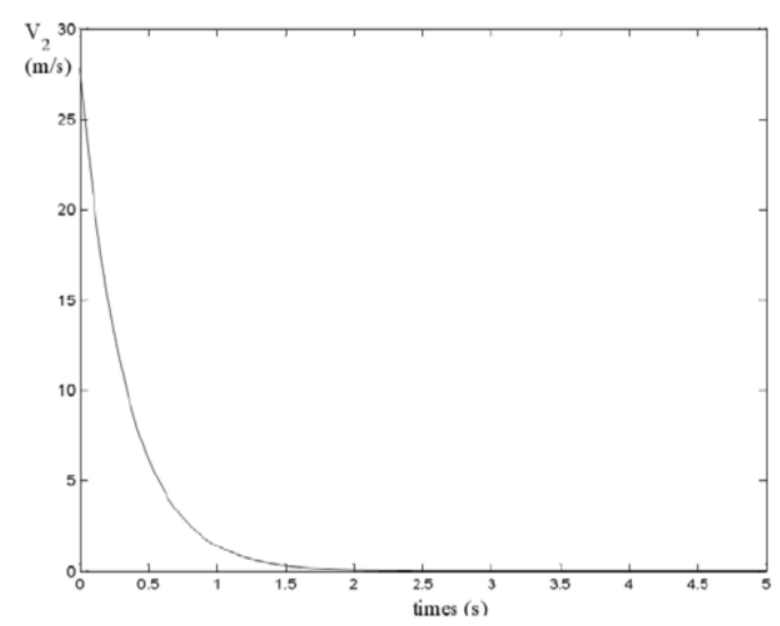

(a)

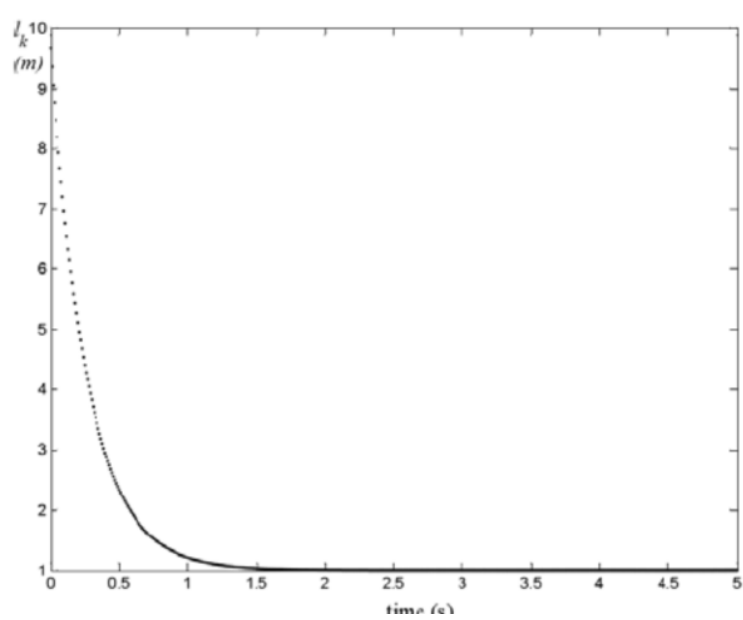

(b)

Figure 3. a) At left, a plot of $V_{2}(t)$ versus time; $b$ ) at right, a plot of safe distance $I_{2}(t)$ from the first car versus time. Note: as $\mathrm{V}_{2}(\mathrm{t})$ approaches $0 \mathrm{~m} / \mathrm{s}, \mathrm{I}_{2}(\mathrm{t})$ approaches $1 \mathrm{~m}$.

$$
U=\left[\begin{array}{c}
\alpha V_{1}(t) \\
0 \\
\vdots \\
0
\end{array}\right]
$$

is the input vector. As you see, we have considered $V_{1}(t)$ as an input to the car system. This is because if the function $V_{1}(t)$ is determined then all $V_{k}(t)$ are known.

Equation (9) is a first order differential matrix equation. Thus it is easy to solve and get answers. One method is to apply the Laplace Transform to both sides of the equation. We will solve (9) using a Matlab computer simulation.

\section{MATLAB SIMULATION}

\section{a. Breaking Process}

We are interested to sketch the wave function $V(k, t)$, as described by equation (4). As a function of the car index $k$. Let us take $V_{1}(t)=0$ for all $t$, meaning that all cars should prepare to break and come to a stop.

Since the first car is stopped, the safe distance $I_{2}(t)$ decreases continuously, and therefore the driver of the second car pushes the brake pedal instantly. Figure 3(a) shows the velocity of the second car, $V_{2}(t)$ versus $t$. Figure $3(b)$ depicts the safe distance $I_{2}(t)$ as a function of time. The values of the parameters used in Figure 3 are gathered together in Table 1 . We will use these values throughout the present analysis. These numbers are obtained from real observations and experiments along the Hemmat Highway in Tehran.

\begin{tabular}{|c|c|}
\hline Parameter & Value \\
\hline$l$ & $10 \mathrm{~m}$ \\
\hline$l^{\prime}$ & $1 \mathrm{~m}$ \\
\hline$V_{0}$ & 200 \\
\hline$n$ & $3.08 \mathrm{sm}^{-1} / \mathrm{h} 28 \mathrm{~m} / \mathrm{s}$ \\
\hline$\alpha=\frac{V_{0}}{l-l \prime}$ & \\
\hline$\tau=\frac{1}{\alpha}$ & $0.324 \mathrm{~s}$ \\
\hline
\end{tabular}

Table 1. Values of parameters. 


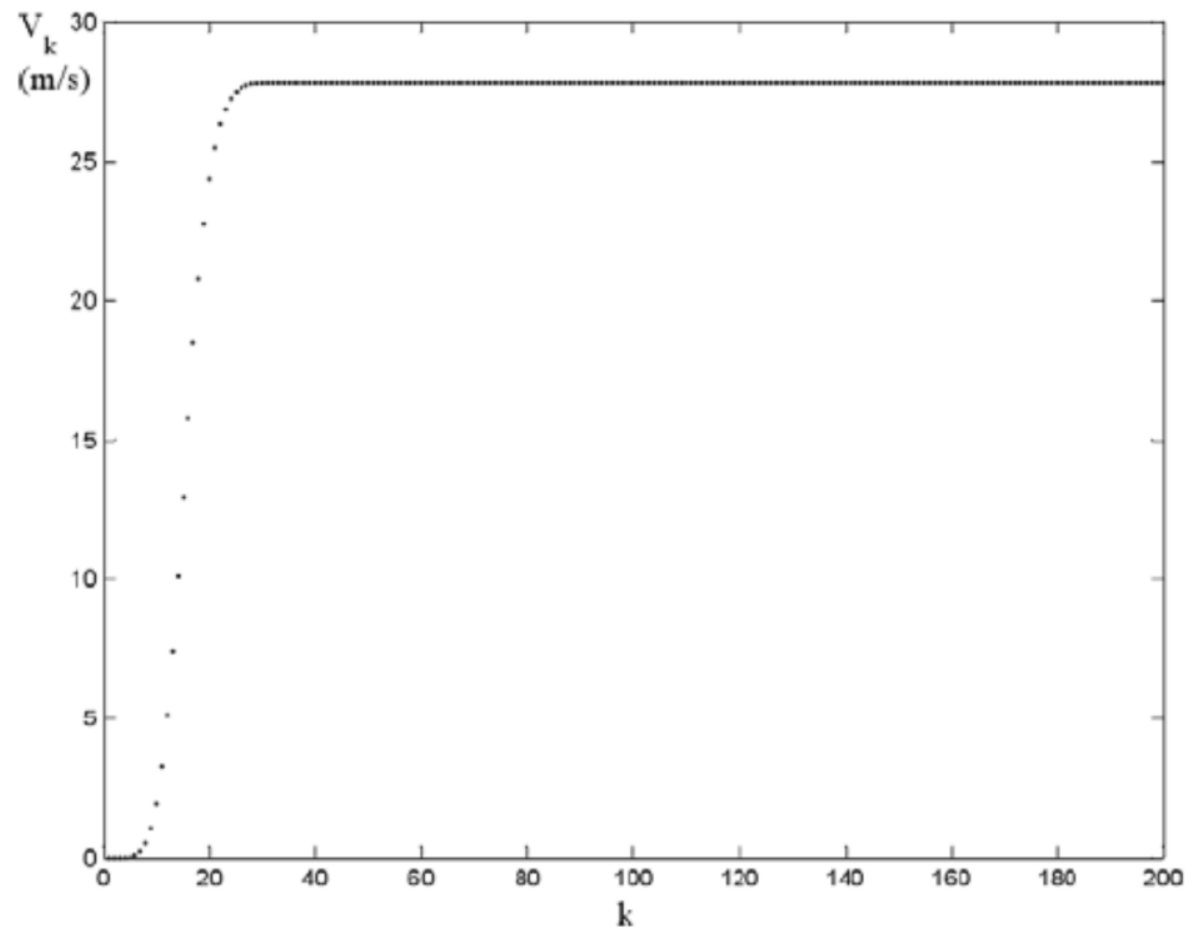

Figure 4. Plot of $V_{k}(t)$ versus $\mathrm{k}$ (index for the cars) at time equal to $5 \mathrm{~s}$. Each dot represents a car.

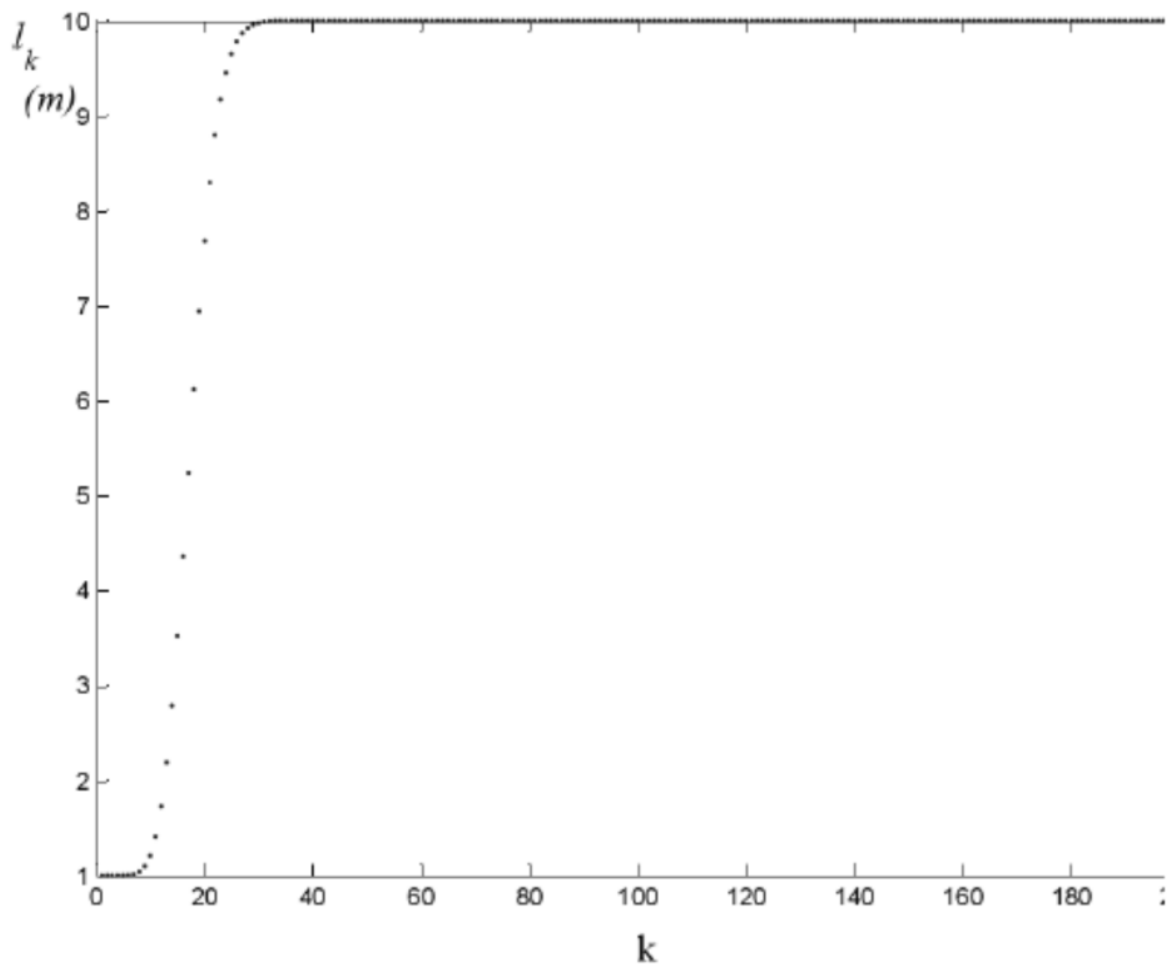

Figure 5. Plot of the safe distance function $I_{k}(t)$ for each of the 200 cars at time equal to $5 \mathrm{~s}$. Dots represent individual cars. 


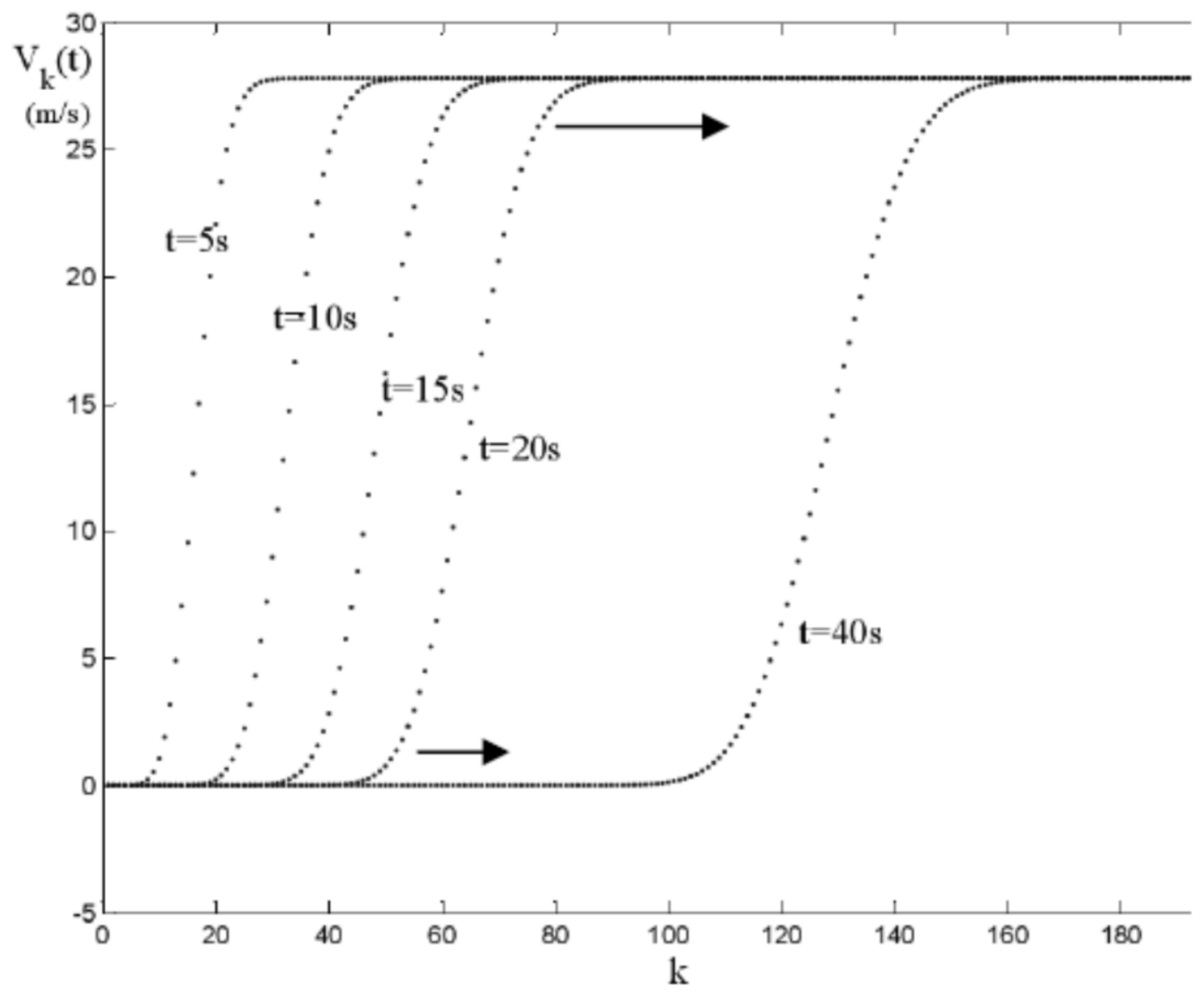

Figure 6. Graph of velocity versus $k$ at different sampling times. We call it the Brake Wave. Numbers on the plots are time values.

Now let us look at the wave function $V(k, t)$. We will model a stretch of highway on which 200 cars are driving. It is useful to illustrate the $V(k, t)$ function versus $\mathrm{k}$, at a specific time. Figure 4 shows the velocity of each car $(k)$ at a fixed time $t=t_{0}$. According to the parameters in Table 1 , at $t=5 \mathrm{~s}$ almost 10 cars have stopped and nearly 20 cars are in the middle of braking preparing to stop. The other 170 cars are driving at the rated speed $V_{0}$.

Figure 5 shows the safe distance function $I_{k}(k)$ for each car at time $t=5 \mathrm{~s}$. As you may notice, this distance is $1 \mathrm{~m}(=l)$ for stationary cars and $10 \mathrm{~m}(=l)$ for cars driving at the rated speed.

Figure 6 depicts the velocity-car curve for different values of sampling time: $5 \mathrm{~s}, 10 \mathrm{~s}, 15 \mathrm{~s}, 20 \mathrm{~s}$ and finally $40 \mathrm{~s}$. It is similar to a moving wave that propagates to the right. Obviously, as the wave propagates to the right, its shape changes a bit. This change is recognized by the change in the number of cars with velocities between 0 and $V_{0}$. This means that the value of $\tau$ calculated in equation (8) depends on t. As Figure 6 shows, this dependency is not strong.

The wave function $V_{k}(t)$ moves with speed $\alpha(\approx 3)$ of about 3 cars per second. However, the upper side of the wave shape moves faster than the lower side. That is, the number of cars preparing to stop increases with time. We will discuss this further in Section IV.

\section{b. Running Process}

In this section, we will assume that all cars are stationary, separated by a distance $I$ '. Therefore all cars initially have $V_{k}(0)=0$. At $t$ $=0$, the first car starts to move and it reaches the rated velocity very quickly. 


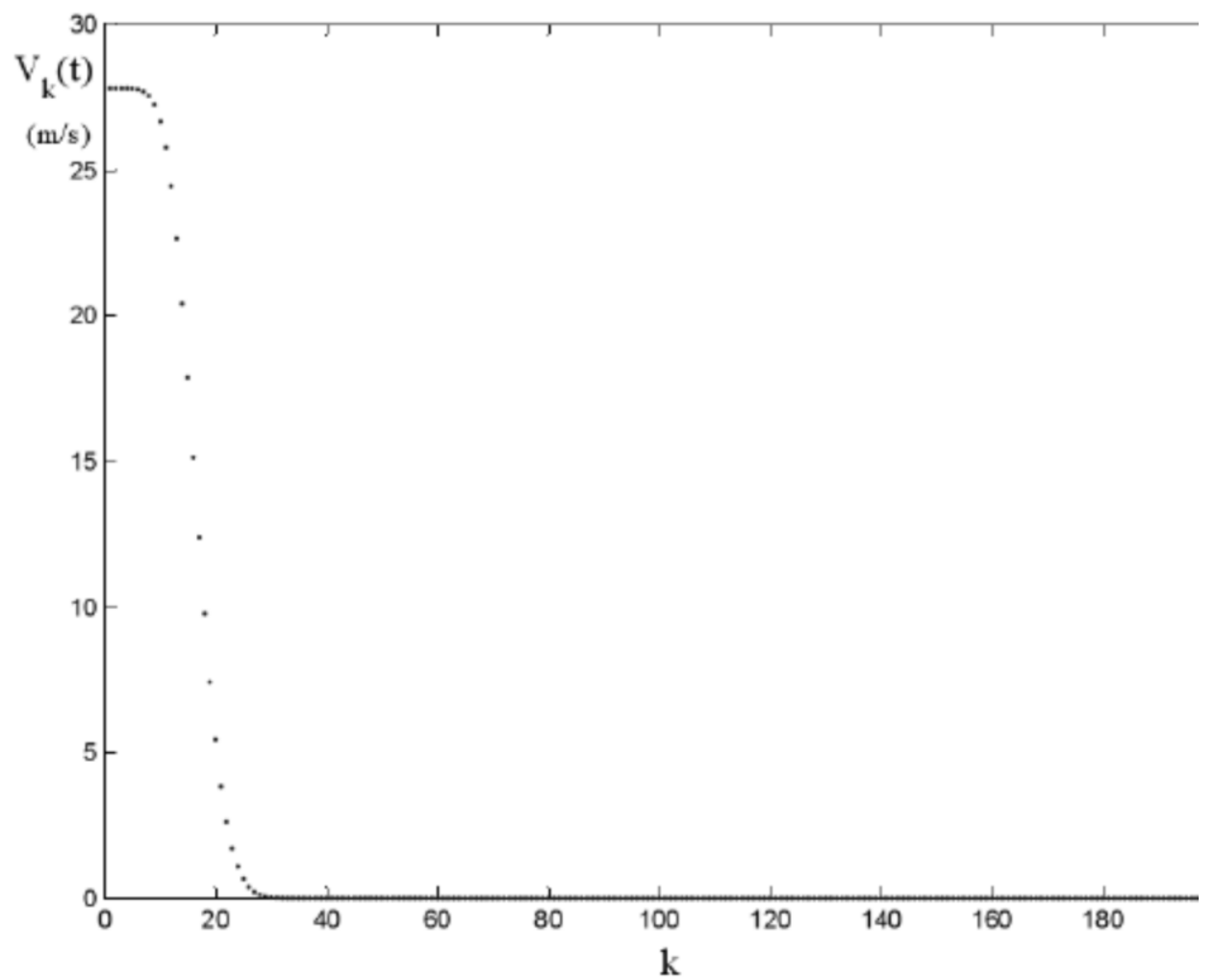

Figure 7. Plot of $V_{k}(t)$ versus $k$ (cars) at time equal to $5 s$ from the start of a process. Each dot represents a car.

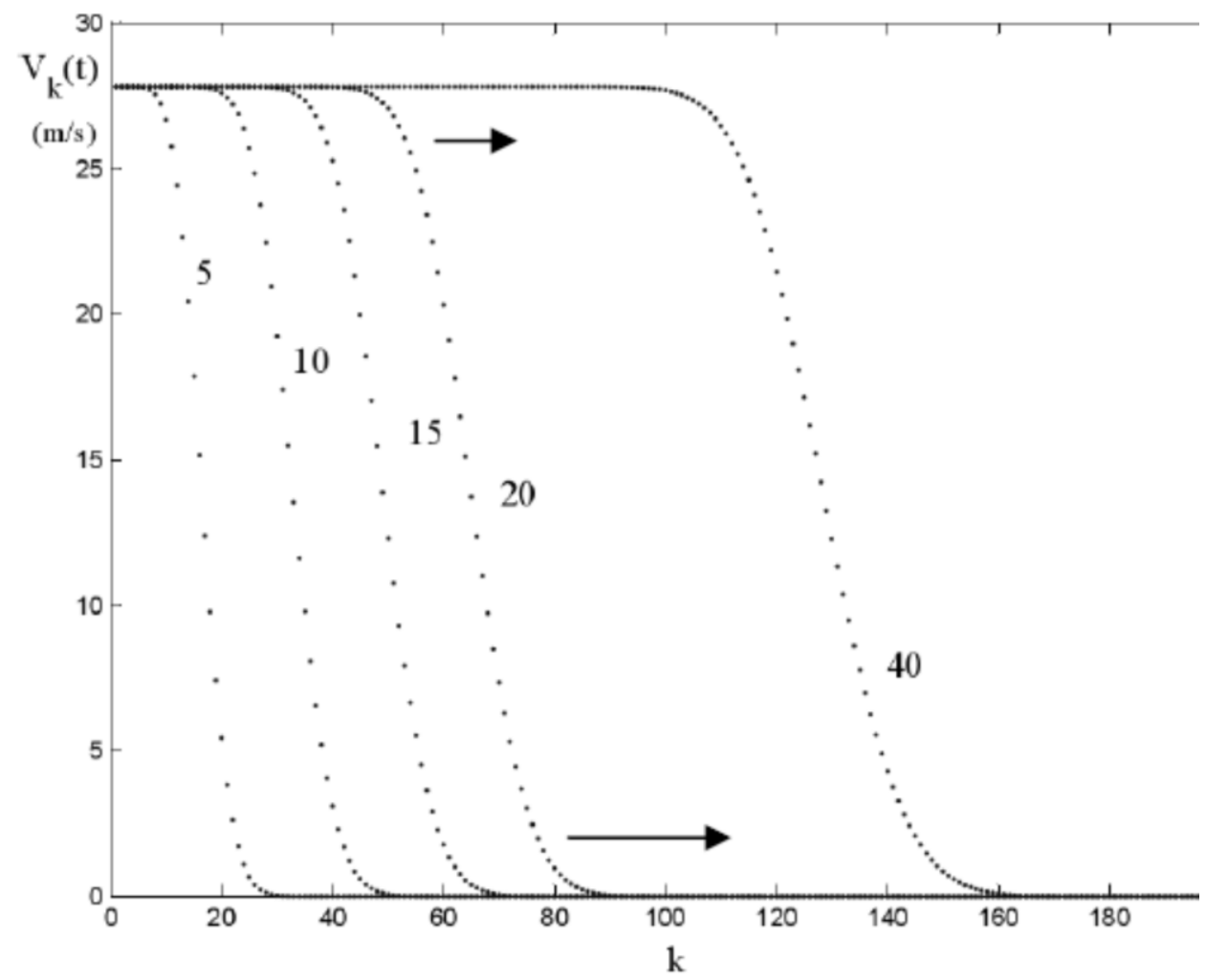

Figure 8. Graphs of velocity versus $k$ at different sampling times. We call it the Run Wave. Numbers written on the plots are time values in seconds. 


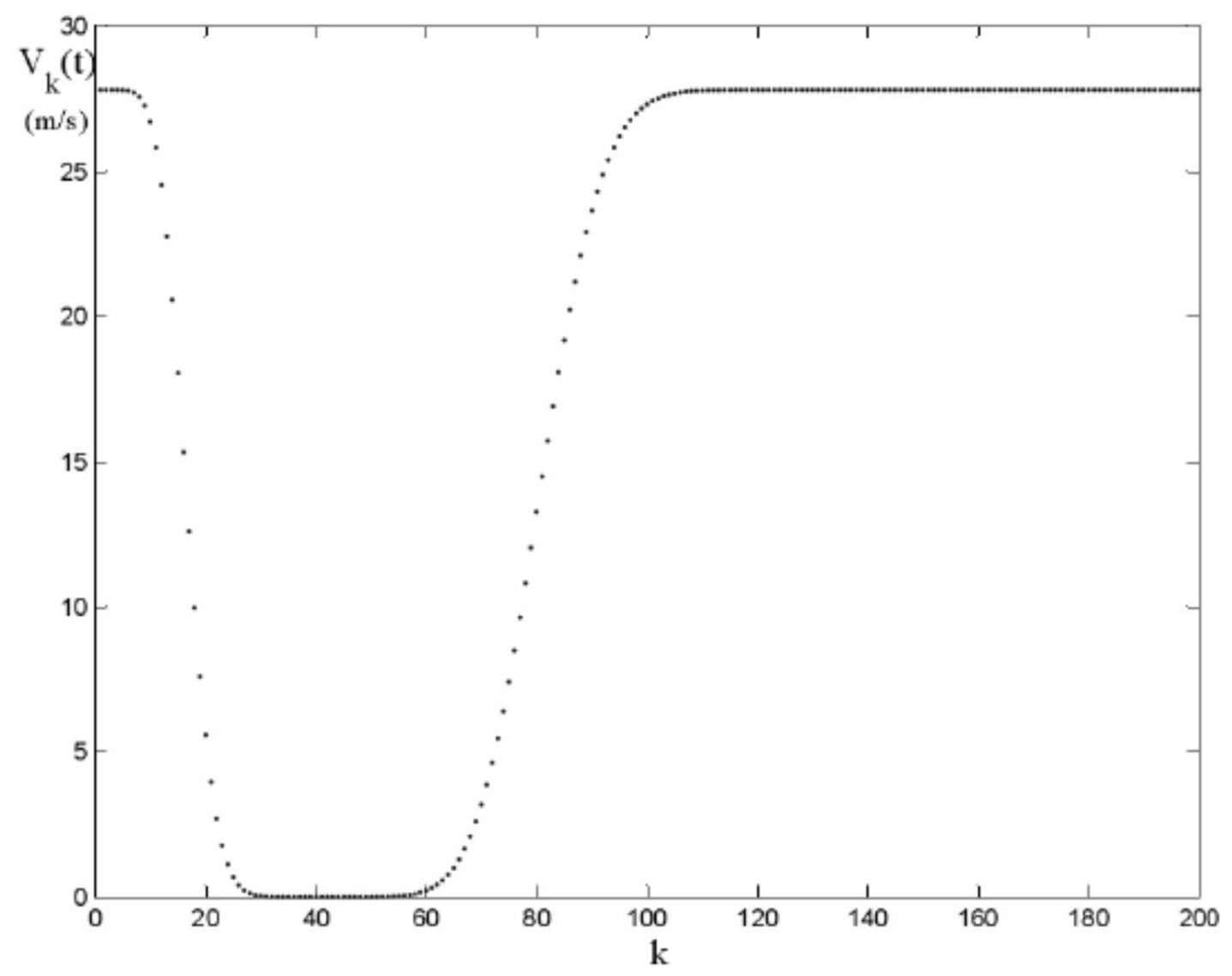

Figure 9. Plot of $V_{k}(t)$ versus $k$ for $T=20 \mathrm{~s}$ at $t=25 \mathrm{~s}$.

We are interested in finding the step response of the car-traffic system. We take the input of the system as

$$
V_{1}(t)=V_{0} \cdot u(t)
$$

Where $u(t)$ is the unit step function. Figure 7 shows the result of running the simulation to check behavior for $t>5 \mathrm{~s}$.

As in Figure 6, we have depicted the moving wave function $V_{k}(t)$ for several time values: $5 \mathrm{~s}, 10 \mathrm{~s}, 15 \mathrm{~s}, 20 \mathrm{~s}$ and $40 \mathrm{~s}$. It is noticeable that the shape of the curve changes as it passes over the cars. That is, the lower part of the wave propagates faster than the upper part.

\section{c. Brake and Run Process: Interruption Phenomenon}

Now let us consider the situation discussed in Section II B (Speed Control Modeling), i.e. the braking process. But this time the first car will continue along its path after a time delay. In this section we will assume that the first car is at rest at $t \leq 0$ and stays at rest until $t=T$ when it begins to move. Therefore the input function for the system is taken to be

$$
V_{1}(t)=V_{0} \cdot u(t-T)
$$

The same as in previous sections, we want to find the step response of the car-traffic system. Figure 9 shows the wave function $V_{k}(t)$ versus $\mathrm{k}$ for $\mathrm{T}=20 \mathrm{~s}$. Figure 9 is similar to Figure 6 apart from the Run process. According to this figure, almost 30 cars are still at rest.

Figure 10 depicts the same plot at $t$ $=45 \mathrm{~s}$. Note that about 7 or 8 cars are at rest. It seems that the Run-wave travels faster than the Brake wave. This should be no surprise: we saw in Figures 6 and 8 that the lower part of the Run wave travels faster than the upper part, and also that the lower 


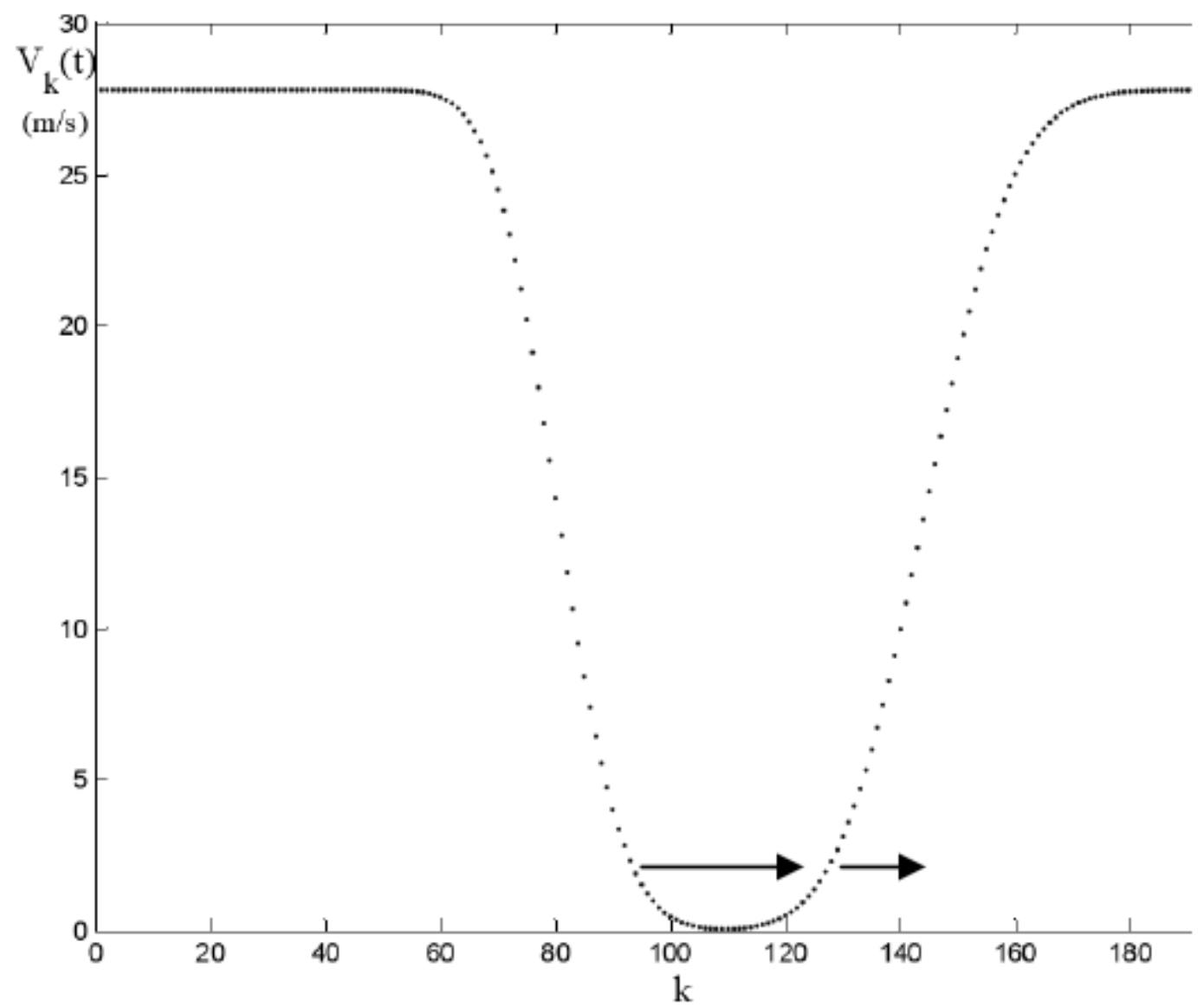

Figure 10. Plot of $V_{k}(t)$ versus $\mathrm{k}$ with $\mathrm{T}=20 \mathrm{~s}$ at $\mathrm{t}=45 \mathrm{~s}$.

part of the Brake wave travels more slowly than the upper part of the wave shape. Since we have symmetry in the problem (the equation of motion is the same), it must be that the lower part of the Run wave moves faster than the Brake wave's lower part. This means that after some characteristic time, no car would stop completely though speed would be reduced. This is observed to be a real-life phenomenon. That is, if the interruption time is $T$ seconds for the first car, it is shorter for the $\mathrm{k}^{\text {th }}$ car and may approach zero for cars far back from the first car. When this happens we say that the information has decayed to wave propagation.

\section{DELAYED STEP RESPONSE}

In this case, we focus on the delayed step response of the system when input is as given in (11), namely

$$
V_{1}(t)=V_{0} \cdot u(t-T)
$$

First of all, let us start with (4) and use equation (5) to find the dependence of $\tau$ (wave period) on both time and $k$. In general $\tau(t, k)$ is a function of both $t$ and $k$. In (6) and (7) we had an approximate determination $\tau$ to be a constant. But now, let us expand equation (6) to second order in $\tau$. Doing this we have,

$$
V_{k}(t+\tau) \approx V_{k}(t)+\frac{d V_{k}(t)}{d t} \tau+\frac{d^{2} V_{k}(t)}{d t^{2}} \frac{\tau^{2}}{2}
$$

Substituting (12) into equation (4) gives, 


$$
\frac{d V_{k}(t)}{d t}=\alpha\left(\frac{d V_{k}(t)}{d t} \tau+\frac{d^{2} V_{k}(t)}{d t^{2}} \frac{\tau^{2}}{2}\right)
$$

Extending our first-order expansion, we can expand $\tau$ to second order to get,

$$
\tau \approx \frac{1}{\alpha}+\frac{\beta}{\alpha^{2}}
$$

Simplifying equation (13) using (14), we get to a new equation in $\tau$.

$$
\frac{1}{2} \frac{d^{2} V_{k}(t)}{d t^{2}}+\beta \frac{d V_{k}(t)}{d t}=0
$$

Therefore,

$$
\tau \approx \frac{1}{\alpha}-\frac{1}{2 \alpha^{2}} \frac{\frac{d^{2} V_{k}(t)}{d t^{2}}}{\frac{d V_{k}(t)}{d t}}
$$

Using equation (13) one more time, we gain a form that may be easier to understand.

$$
\tau \approx \frac{1}{2 \alpha}\left(3-\frac{\frac{d V_{k-1}(t)}{d t}}{\frac{d V_{k}(t)}{d t}}\right)
$$

If you focus on the lower part of the Brake wave graph (Figure 6), the magnitude of the $k^{\text {th }}$ car's acceleration is more than that of the (k-1) car. In contrast, at the upper end of the Brake wave, the acceleration magnitude of the $k^{\text {th }}$ car than that of car $(k-1)$. Thus, according to equation (17), the Brake wave propagates faster at the upper side.

From symmetry, in the Running wave (Figure 8) the lower part travels faster than the upper side (see Figure 10). Thus there is an interference, and the two waves (Brake and Run) go through each other at the lower side of the wave shape.

\section{FLOW RATE AND DENSITY FUNCTION}

Let us define $q(x, t)$ as a flow rate of the cars, that is, $q(x, t)$ is the number of cars per unit time passing a given point $x$ at time $t$ (a flux). Therefore,

$$
q(x, t)=\frac{V_{k}(t)}{l_{k}(t)}
$$

Using equation (1) to substitute for $V_{k}(t)$,

$$
q(x, t)=\frac{V_{0}-\alpha l}{l_{k}(t)}+\alpha
$$

Also, we can define the numbers of cars per unit length of highway as a function of location and time:

$$
\rho(x, t)=\frac{1}{l_{k}(t)}
$$

Joining equations (19) and (20) we get an equation relating flow rate and density:

$$
q=\left(V_{0}-\alpha l\right) \rho+\alpha
$$

If we assume that the maximum allowable speed is $V_{0}$ then for $I_{k}(t)>I$, equation (21) reduces to

$$
q=\rho V_{0}
$$

Figure 11 displays both equations (21) and (22).

The behavior seen in Figure 11 is similar to what is seen in the relationship between flow and density on highways. That is, the flow increases to a maximum value and then begins to decrease with a to a lower rate $[5,6]$.

\section{CONCLUSION}

We have presented a microscopic model for traffic flow that shows how macroscopic kinematics emerge from the behaviors of individual cars. Traffic flow has been modeled from the perspective of the individual driver, making it well suited for simulation.

The traffic wave was investigated and plotted using Matlabß 7.6. The group velocity of the wave was determined using an equation of motion, and we have shown 


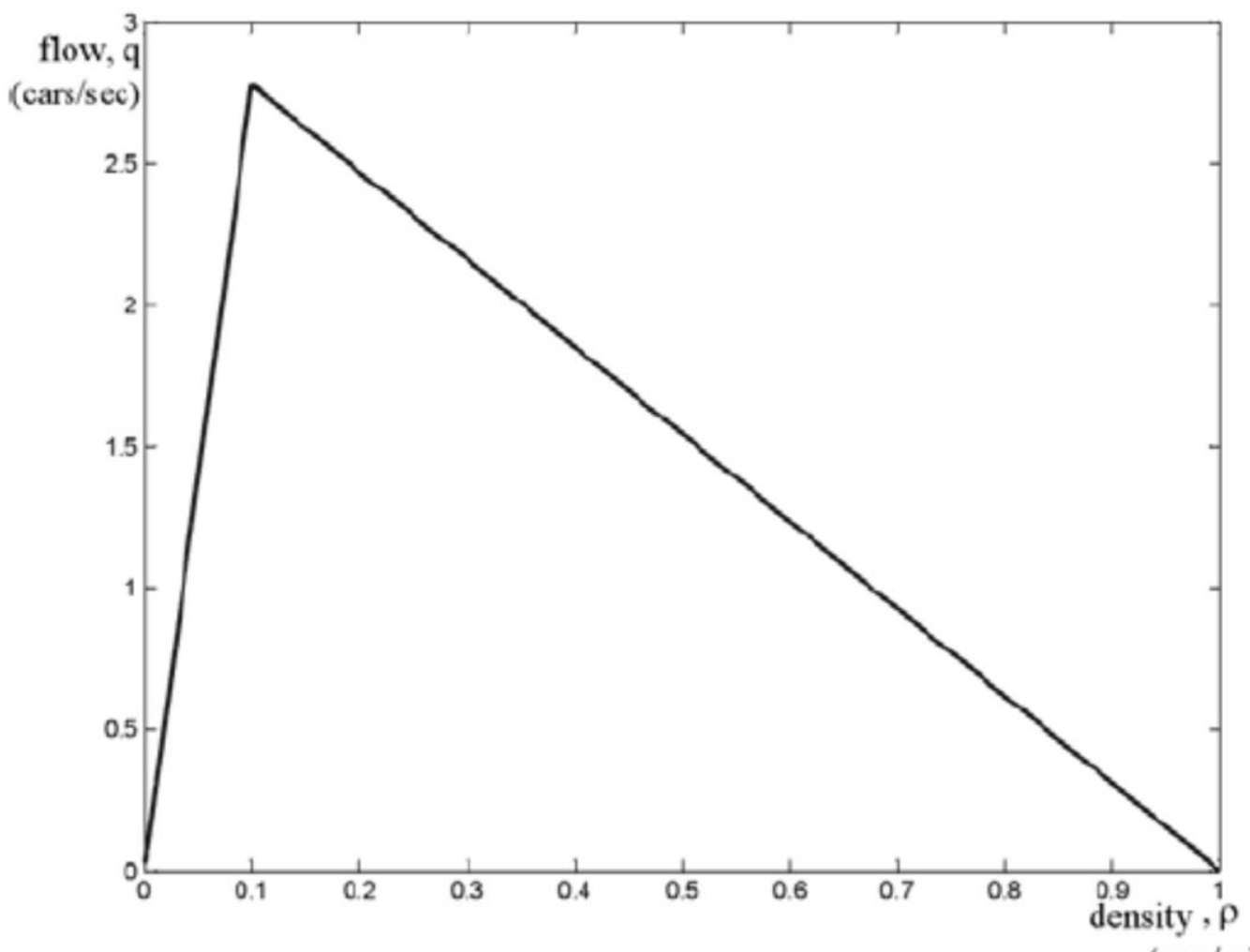

Figure 11. Relationship between flow and density along the highway.

that the group velocity differs from side to side of the wave shape.

We introduced a new input to the traffic model: the velocity of each car depends on a feedback of the distance to the car in front of it, with a constant "set point" I.

\section{REFERENCES}

1. M. J. Herry, "Principles of Physics Applied to Traffic Movements and Road Conditions," American Journal of Physics, 13(1) (1945).

2. Debashish Chowdhury, Ludger Santen, Andreas Schadschneider, "Statistical Physics of Vehicular Traffic and Some
Related Systems," Physics Reports, 329 (2000).

3. Dirk Helbing and Benno Tilch, "Generalized Force Model of Traffic Dynamics," Physics Review E, 58(1) (1998).

4. N. O. Hodas and A. Jagota, "Microscopic Modeling of Multi-lane Highway Traffic Flow," American Journal of Physics, 71 (12), (December 2003).

5. J. N. Johnson, "Kinematic Waves and Group Velocity," American Journal of Physics, 42 (1974) pp. 681-688.

6. Dirk Helbing, "Traffic and Related SelfDriven Many Particle Systems," Reviews of Modern Physics, 74(4) (2001) p. 1108. 